Research Paper

\title{
Clinicopathological and prognostic significance of programmed death ligand 1 expression in Korean melanoma patients
}

\author{
Sumi Yun 1 , Yujun Park ${ }^{2}$, Seyoung Moon², Soomin Ahn², Kyoungyul Lee ${ }^{3}$, Hyo Jin Park ${ }^{4}$, Hye Seung Lee², \\ Gheeyoung Choe ${ }^{2}$, Kyu Sang Lee ${ }^{2 \bowtie}$ \\ 1. Department of Diagnostic Pathology, Samkwang Medical Laboratories, Seoul, Republic of Korea \\ 2. Department of Pathology, Seoul National University Bundang Hospital, Seongnam-si, Gyeonggi-do, Republic of Korea \\ 3. Department of Pathology, Kangwon National University Hospital, Chuncheon-Si, Kangwon-Do, Republic of Korea \\ 4. Department of Pathology, Sheikh Khalifa Specialty Hospital, Ras al Khaimah, United Arab Emirates \\ $\triangle$ Corresponding author: Kyu Sang Lee, MD, PhD, Department of Pathology, Seoul National University Bundang Hospital, 173-82 Gumi-ro, Bundang-gu, \\ Seongnam-si, Gyeonggi-do 463-707, Republic of Korea. Tel: +82 31787 7716; e-mail: tigerkyu@gmail.com \\ (c) Ivyspring International Publisher. This is an open access article distributed under the terms of the Creative Commons Attribution (CC BY-NC) license \\ (https://creativecommons.org/licenses/by-nc/4.0/). See http://ivyspring.com/terms for full terms and conditions.
}

Received: 2018.10.11; Accepted: 2019.05.03; Published: 2019.06.02

\begin{abstract}
Programmed death ligand 1 (PD-LI) expression provides significant value to predict prognosis and response following immunotherapy in several types of cancers. However, its clinicopathological and prognostic significance in melanoma remains unclear. PD-LI and the number of tumor infiltrating lymphocytes (TILs) were investigated in 63 Korean patients with melanoma based on the melanoma scoring system. We also compared the results using the PD-LI antibodies-22C3 and EIL3N clones. In addition, BRAF gene mutation was detected using anti-BRAF antibody and real-time polymerase chain reaction. Overall, 29 (46.0\%), 16 (25.4\%), and 18 (28.6\%) patients exhibited the acral lentiginous type, nodular type, and other histological subtypes of melanoma, respectively. PD-L1 expression was detected in $37(58.7 \%)$ cases and was closely associated with a CD8+TIL high phenotype $(P<0.001)$. Combined survival analysis depending on PD-LI and CD8+TILs status showed that the PD-L1-/CD8+TIL high group demonstrated the best survival outcome, whereas patients with $\mathrm{PD}-\mathrm{LI}+/ \mathrm{CD} 8+\mathrm{TIL}{ }^{\text {low }}$ showed the worst prognosis $(P=0.039)$. However, PD-L1+/CD8+ TIL low was not an independent prognostic factor. The 22C3 and ElL3N clones showed a high concordance rate (kappa value, 0.799 ). BRAF mutation status was not correlated with PD-L1 expression. We suggest that evaluation of the combined status of PD-LI and TIL might be useful to predict the survival of patients with melanoma.
\end{abstract}

Key words: melanoma, prognosis, programmed death ligand 1, tumor infiltrating lymphocytes, 22C3

\section{Introduction}

Melanoma is a highly malignant skin tumor and a leading cause of cancer-related mortality in Western countries, ranking as the fifth most common cancer in the USA [1, 2]. The incidence and pathogenesis of melanoma both show variation according to ethnicity [3], with a lower incidence of melanoma in Asians than in Caucasians, and the predominant location of the tumor also differs according to race [4].

Melanoma is classified into several histological subtypes associated with various molecular and clinicopathological characteristics [5]. The most common types of melanoma in Caucasians are found on sun-exposed portions of the skin, such as nodular melanoma and superficial spreading melanoma. In contrast, acral lentiginous melanoma that occurs on non-sun damaged areas is the most common subtype detected in Asians [6, 7]. Chronic sun-damaged forms of melanomas are associated with NRAS, BRAF non-V600E, or KIT mutations, whereas non-sun-damaged melanomas are associated with a 
predominance of BRAF V600E mutations [8]. Therefore, research on melanoma must take variations among races and subtypes into consideration.

Several multi-institutional clinical trials have indicated a survival benefit of programmed death 1 (PD-1)/ programmed death ligand 1 (PD-L1) blocking agents in patients with melanoma [9, 10]. Accordingly, anti-PD-1/PD-L1 therapy has recently become the most important melanoma treatment. The United States Food and Drug Administration (FDA) approved the anti-PD-1/PD-L1 drug nivolumab as the first-line treatment for patients with $B R A F$ wild-type advanced melanoma [11, 12]. PD-1 is an immune inhibitory receptor expressed on activated lymphocytes $[13,14]$, and interaction with its ligands PD-L1 and PD-L2, which are expressed in both tumor cells (TCs) and immune cells, plays a pivotal role in the tumor's ability to escape from immune attack [14]. Hence, inhibition of the PD-1/PD-L1 axis can be a powerful therapeutic strategy to promote the immune response to invading cancer cells.

Accordingly, detection of PD-L1 expression is an important factor in the decision for administering a PD-1/PD-L1 inhibitor to patients with several types of cancers. The FDA approved PD-L1 immunohistochemistry (IHC) as a companion or complementary diagnostic tool for PD-1/PD-L1 inhibitor therapy in patients with non-small cell lung cancer or urinary bladder cancer. However, currently, PD-1/PD-L1 inhibitors are used in the treatment of melanoma regardless of the specific PD-L1 expression in patients.

Furthermore, PD-L1 expression itself was reported to be significantly correlated with an unfavorable prognosis in various malignancies, including non-small cell lung cancer, colorectal cancer, renal cell carcinoma, and breast cancer [10, 15-17]. However, the prognostic value of PD-L1 expression status in melanoma is controversial [10, 18]. Several recent studies have indicated the prognostic significance of PD-L1 expression in not only TCs but also in tumor infiltrating lymphocytes (TILs) $[19,20]$.

Even if PD-L1 expression is not correlated with the response to PD-1/PD-L1 inhibitors in melanoma patients, it is still necessary to precisely assess the potential clinical role of PD-L1 expression in melanoma. Therefore, in the present study, we evaluated the PD-L1 status in Korean melanoma patients using an FDA-approved antibody (22C3; Dako, Carpentaria, CA, USA) as well as another commercially available PD-L1 antibody (E1L3N; 1:50, Cell Signaling Technology, Danvers, MA, USA). The PD-L1 expression status was quantified using a scoring system specific to melanoma with relation to its expression on both TCs and/or TILs [21].

\section{Materials and Methods}

\section{Tissue samples and tissue microarray (TMA) construction}

A total of 63 patients diagnosed with malignant melanoma from March 2006 to February 2013 at Seoul National University Bundang Hospital were enrolled in this study. Thirty-six (57.1\%) patients underwent surgical excision and $27(42.9 \%)$ patients underwent punch biopsy. All cases were classified based on histologic type such as acral lentiginous, nodular, and other melanoma subtypes. Patients who had preoperative chemo-radiation therapy were excluded from the study. Clinical information and pathologic characteristics were compiled from medical and pathologic records. The assessment of clinical nodal and metastasis stage was determined in 58 cases based on the radiologic and pathologic evaluation. The clinical stage could not be determined for seven cases due to follow-up loss and refusal of further radiologic evaluation.

For TMA construction, we selected representative regions of tumors, and core samples with a 2-mm diameter were transferred to recipient blocks and re-embedded, as described previously (SuperBioChips Laboratories, Seoul, South Korea) [22]. In primary lesion, we selected the tumor area with the invasive border for including TILs. In addition, considering that tumor reactive immune cells infiltrate the metastatic melanoma via vascular endothelial cells, we obtained tissue from the tumor center with intratumoral blood vessels for the metastatic lesion.

This study was approved by the Institutional Review Board of Seoul National University Bundang Hospital (IRB number: B-1807-481-302). For this type of study, formal consent was not required.

\section{Immunohistochemistry and scoring}

IHC staining was performed on the TMA slides using a PD-L1 clone (22C3; Dako, Carpentaria, CA, USA) and stained with Dako Autostainer (Dako) (Figure 1). IHC was also performed with an additional PD-L1 antibody (E1L3N; 1:50, Cell Signaling Technology, Danvers, MA, USA) and CD8 (1:100, Dako, Glostrup, Denmark) on the Ventana Benchmark XT system (Ventana, Tucson, AZ, USA), and visualized with a DAB detection kit (Ventana).

CD8+ TILs were evaluated in at least 3-4 representative high-power field (HPF) areas from each sample, and the mean CD8+ TILs count was scored using the following four-tier scoring system: 
no lymphocytes (0), 1-10/HPF (1), 11-50/HPF (2), $>50 / \mathrm{HPF}$ (3) [23].

We evaluated the PD-L1 expression in TCs or TILs separately, and then in both TC and / or TIL. The PD-L1 positivity was categorized based on the melanoma scoring system (MEL) as reported by Daud et al. [21]. The membranous expression in PD-L1 was graded on a five-point scale as follows: 0, no membranous staining; $1,>0 \%-<1 \% ; 2, \geq 1 \%-<10 \% ; 3$, $\geq 10 \%-<33 \% ; 4, \geq 33 \%-<66 \% ; 5, \geq 66 \%$.

\section{BRAF mutation analysis}

$B R A F$ gene mutation status was determined by IHC staining with anti-BRAF antibody (Ventana, Tucson, AZ, USA) and real-time polymerase chain reaction (PCR). Samples from all 63 patients were subjected to BRAF IHC. For real-time PCR analysis, DNA was extracted from the tissues of 40 melanoma patients, including 15 BRAF IHC-positive, 20 BRAF IHC-negative, and five equivocal cases, using Cobas DNA Sample Preparation Kit (Roche, Basel, Switzerland), according to the manufacturer's instructions and as described previously [24]. Samples were then screened for the BRAF (V600E) mutation using Cobas 4800 System (Roche).

\section{Statistical analysis}

All statistical analyses were performed with SPSS Statistics 21.0 (IBM Corp., Armonk, NY, USA). The correlation between PD-L1 expression and clinicopathological variables was analyzed with the $\chi^{2}$ test and Fisher's exact test. Survival differences were estimated by the Kaplan-Meier method with the log-rank test. Multivariate analysis was performed using Cox proportional hazards regression model. A $P$-value of $<0.05$ was considered statistically significant. The concordance of PD-L1 expression evaluated using two different antibodies (22C3 and E1L3N) was determined using Cohen's kappa test.

\section{Results}

\section{Patient characteristics}

The patients' clinicopathological characteristics are summarized in Table 1 . The patient cohort consisted of $27(42.9 \%)$ men and $36(57.1 \%)$ women. The median age of the patients was 65 years (range, 25-90 years). The histologic subtypes included 29 $(46.0 \%)$ acral lentiginous, 16 (25.4\%) nodular, and 18 (28.6\%) other melanoma types such as superficial spreading melanoma, lentigo maligna, and

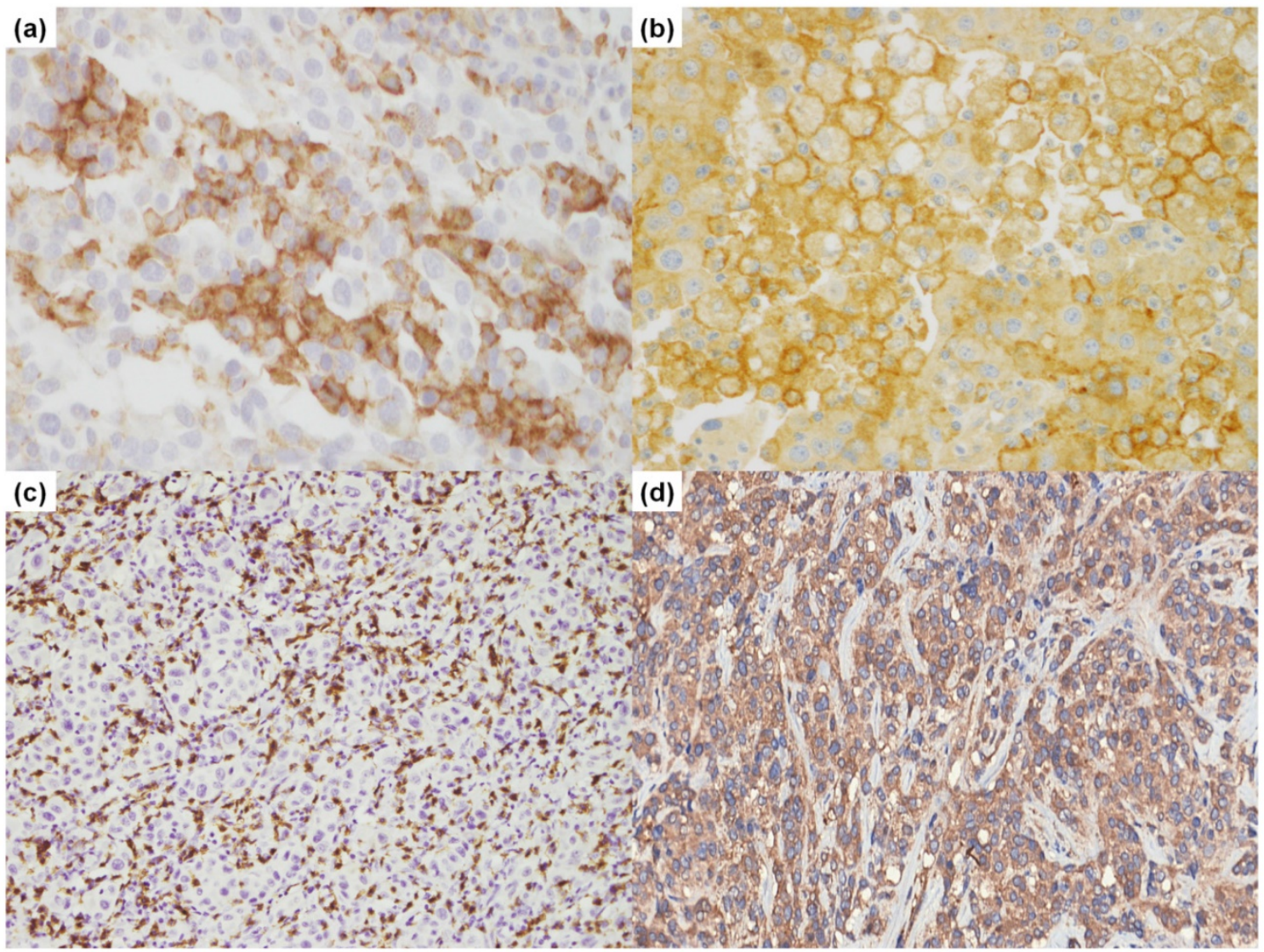

Figure 1. Immunohistochemical results of programmed death ligand 1 (PD-L1) and CD8. (a). PD-L1 (22C3, 400x); (b). PD-L1 (E1L3N, 400x); (c). CD8 (200x); (d). BRAF (400x). 
unclassified type. The follow-up time ranged from 0.4 to 127.6 months and the median follow up time was 31.5 months. In present study, no patients received neoadjuvant chemotherapy. Fourteen patients received postoperative chemotherapy; however, unfortunately no patients received anti PD-1/PD-L1 therapy. Fifteen patients received radiotherapy.

Table 1. Patients' clinicopathologic characteristics

\begin{tabular}{ll}
\hline Characteristics & No $(\%)$ \\
\hline Total & 63 \\
Age, median (range) & $65(25-90)$ \\
Sex & \\
Male & $27(42.9 \%)$ \\
Female & $36(57.1 \%)$ \\
Melanoma subtype & \\
Acral lentiginous & $29(46.0 \%)$ \\
Nodular & $16(25.4 \%)$ \\
Other subtype & $18(28.6 \%)$ \\
cN stage (n= 58) & \\
No & $43(74.1 \%)$ \\
N+ & $15(25.9 \%)$ \\
cM stage (n = 58) & \\
M0 & $47(81.0 \%)$ \\
M+ & $11(19.0 \%)$ \\
Lymphovascular invasion & \\
Absent & $56(88.9 \%)$ \\
Present & $7(11.1 \%)$ \\
Ulceration & \\
Absent & $20(31.7 \%)$ \\
Present & $11(17.5 \%)$ \\
Not assessed & $32(50.8 \%)$ \\
Breslow thickness & \\
$<1$ mm & $9(14.3 \%)$ \\
$\geq 1 m m$ & $26(41.3 \%)$ \\
Not assessed & $28(44.4 \%)$ \\
Specimen site & \\
Primary lesion & $51(81.0 \%)$ \\
Metastatic lesion & $12(19.0 \%)$ \\
Type of surgery & \\
Punch biopsy & $27(42.9 \%)$ \\
Excisional biopsy & $36(57.1 \%)$ \\
Absent & \\
Present & $48(76.2 \%)$ \\
\hline & $15(23.8 \%)$ \\
\hline &
\end{tabular}

\section{PD-LI expression in TCs and TILs}

PD-L1 (22C3) expression in TCs and TILs were found in $32(50.8 \%)$ and $36(57.1 \%)$ cases, respectively (Figure 2). In PD-L1 (22C3) positive subset in TCs, the high expression and low expression groups comprised $6(9.5 \%)$ and $26(41.3 \%)$ cases, respectively. Of the cases with PD-L1 (22C3) expression in TILs, 6 $(9.5 \%)$ and $30(47.6 \%)$ were categorized as high and low expression groups, respectively. Positive PD-L1 (22C3) expression in TCs were more frequently detected in non-acral type $(P=0.017)$ and its expression in TILs also showed a similar trend; however, it was not statistically significant $(P=0.068)$. PD-L1 (22C3) expression in TCs or TILs did not show a correlation with other clinicopathologic variables (Table S1). Using PD-L1 (E1L3N) antibody, 35 (55.6\%) and $38(60.3 \%)$ cases were found to be positive in TCs and TILs, respectively (Figure 2). Among these, PD-L1 (E1L3N) expression in TCs was considered as high expression in $5(7.9 \%)$ cases and $3(4.8 \%)$ cases showed high expression of PD-L1 (E1L3N) in TILs. Similar to PD-L1 (22C3) expression in TCs and TILs, PD-L1 (E1L3N) expression in TCs and TILs was associated with non-acral type $(P=0.037$ and $P=0.020$, respectively) (Table S2). Additionally, we compared the results of PD-L1 expression in TCs or TILs using two antibodies (22C3 and E1L3N), and the results obtained showed moderate agreement.

\section{Association between PD-L1 expression (MEL scoring system) and clinicopathological characteristics}

We assessed the PD-L1 expression in TCs as well as TILs using MEL scoring system. PD-L1 (22C3) positivity was detected in $37(58.7 \%)$ cases, and PD-L1 was highly expressed in seven cases $(11.1 \%)$. The scores of CD8+TILs were divided into low (0-1; CD8+TIL $\left.{ }^{\text {low }}\right)$ and high (2-3; CD8+TIL high) expression, with $45(71.4 \%)$ cases showing the CD8+TIL high phenotype (Figure 2d). According to the histologic subtype, patients with other subtypes showed the highest rate of PD-L1 (22C3) positivity ( $\mathrm{n}=13,76.5 \%)$, followed by those with the nodular type $(\mathrm{n}=11$, $64.7 \%)$ and the acral lentiginous type ( $\mathrm{n}=13,44.8 \%)$. When grouping the patients into acral and non-acral types, PD-L1 (22C3) positivity was more frequently found in the non-acral subtype tumors $(P=0.038)$. Moreover, PD-L1 (22C3) positivity was significantly more frequent in the CD8+TIL high group than in the CD8+TIL low group $(P<0.001)$.

All 15 BRAF IHC-positive cases showed the BRAF (V600E) mutation, whereas the 20 BRAF IHC-negative and five equivocal cases showed the BRAF (V600E) wild type. However, there was no significant association between PD-L1 (22C3) expression status and $B R A F$ mutation status, or other various clinicopathological factors (Table 2).

To accurately validate the PD-L1 detection, we additionally carried out the study using another PD-L1 antibody (E1L3N), which was expressed in 41 $(65.1 \%)$ cases, and five $(7.9 \%)$ cases showed high expression (MEL 4-5) (Table 2). Therefore, use of the PD-L1 (E1L3N) antibody resulted in a higher expression rate as compared to that detected with PD-L1 (22C3); however, high expression (MEL 4-5) was slightly less frequent. Despite these differences, the E1L3N clone showed a good concordance level with the 22C3 clone (kappa value, 0.799; $P<0.001$ ) (Figure 2). The expression of PD-L1 (E1L3N) was also closely associated with the non-acral subtype $(P=$ 0.010) and CD8+TIL high $(P<0.001)$ (Table 1$)$. 

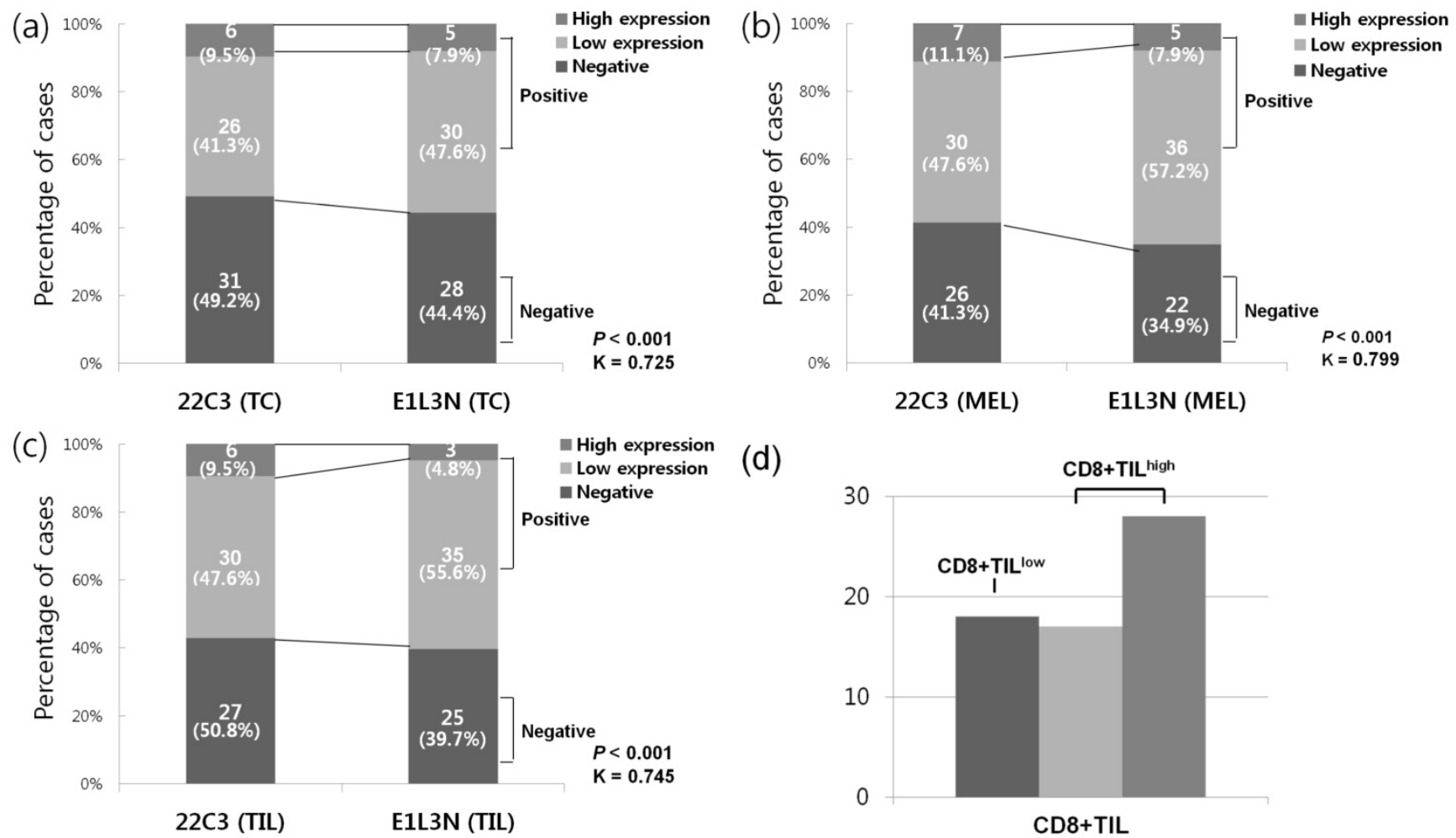

Figure 2. Comparative analysis of programmed death ligand 1 (PD-L1) expression using 22C3 and E1 L3N antibodies, (a) PD-L1 positivity in tumor cells (TCs), (b) PD-L1 positivity in tumor infiltrating lymphocytes (TILs), (c) PD-L1 positivity based on MEL, (d) frequency of CD8+ TIL

Table 2. Association between PD-L1 expression (MEL) and clinicopathologic variables

\begin{tabular}{|c|c|c|c|c|c|c|}
\hline \multirow[t]{2}{*}{ Parameters } & \multicolumn{3}{|c|}{ PD-L1 (22C3) } & \multicolumn{3}{|c|}{ PD-L1 (E1L3N) } \\
\hline & Negative & Positive & $P$ & Negative & Positive & $P$ \\
\hline Age & & & 0.423 & & & 0.595 \\
\hline$\leq 60$ & $11(47.8 \%)$ & $12(52.2 \%)$ & & $9(39.1 \%)$ & $14(60.9 \%)$ & \\
\hline$>60$ & $15(37.5 \%)$ & $25(62.5 \%)$ & & $13(32.5 \%)$ & $27(67.5 \%)$ & \\
\hline Sex & & & 0.555 & & & 0.819 \\
\hline Male & $10(37.0 \%)$ & $17(63.0 \%)$ & & $9(33.3 \%)$ & $18(66.7 \%)$ & \\
\hline Female & $16(44.4 \%)$ & $20(55.6 \%)$ & & $13(36.1 \%)$ & $23(63.9 \%)$ & \\
\hline Melanoma subtype & & & 0.038 & & & 0.010 \\
\hline Acral & 16 (55.2\%) & $13(44.8 \%)$ & & $15(51.7 \%)$ & $14(48.3 \%)$ & \\
\hline Non-acral & $10(29.4 \%)$ & $24(70.6 \%)$ & & $7(20.6 \%)$ & $27(79.4 \%)$ & \\
\hline cN stage $(\mathrm{n}=58)$ & & & 0.723 & & & 0.756 \\
\hline No & $15(34.9 \%)$ & $28(65.1 \%)$ & & $14(32.6 \%)$ & $29(67.4 \%)$ & \\
\hline $\mathrm{N}+$ & $6(40.0 \%)$ & $9(60.0 \%)$ & & $4(26.7 \%)$ & $11(73.3 \%)$ & \\
\hline cM stage $(\mathrm{n}=58)$ & & & 1.000 & & & 0.474 \\
\hline Mo & $17(36.2 \%)$ & $30(63.8 \%)$ & & $16(34.0 \%)$ & $31(66.0 \%)$ & \\
\hline $\mathrm{M}+$ & $4(36.4 \%)$ & $7(63.6 \%)$ & & $2(18.2 \%)$ & $9(81.8 \%)$ & \\
\hline Lymphovascular invasion & & & 0.434 & & & 1.000 \\
\hline Absent & $22(39.3 \%)$ & $34(60.7 \%)$ & & $20(35.7 \%)$ & $36(64.3 \%)$ & \\
\hline Present & $4(57.1 \%)$ & $3(42.9 \%)$ & & $2(28.6 \%)$ & $56(71.4 \%)$ & \\
\hline Ulceration $(\mathrm{n}=31)$ & & & 0.275 & & & 0.262 \\
\hline Absent & $10(50.0 \%)$ & $10(50.0 \%)$ & & $8(40.0 \%)$ & $12(60.0 \%)$ & \\
\hline Present & $3(27.3 \%)$ & $8(72.7 \%)$ & & $2(18.2 \%)$ & $9(81.8 \%)$ & \\
\hline Breslow thickness $(\mathrm{n}=35)$ & & & 1.000 & & & 0.396 \\
\hline$<1 \mathrm{~mm}$ & $3(33.3 \%)$ & $6(66.7 \%)$ & & $3(33.3 \%)$ & $6(66.7 \%)$ & \\
\hline$\geq 1 \mathrm{~mm}$ & $8(30.8 \%)$ & $18(69.2 \%)$ & & $5(19.2 \%)$ & $21(80.8 \%)$ & \\
\hline BRAF mutation & & & 0.909 & & & 0.883 \\
\hline Absent & $20(41.7 \%)$ & $28(58.3 \%)$ & & $17(35.4 \%)$ & $31(64.6 \%)$ & \\
\hline Present & $6(40.0 \%)$ & $9(60.0 \%)$ & & $5(33.3 \%)$ & $10(66.7 \%)$ & \\
\hline CD8+ TIL & & & $<0.001$ & & & $<0.001$ \\
\hline CD8+TIL low & $14(77.8 \%)$ & $4(22.2 \%)$ & & $14(77.8 \%)$ & $4(22.2 \%)$ & \\
\hline CD8+TIL high & $12(26.7 \%)$ & $33(73.3 \%)$ & & $8(17.8 \%)$ & $37(82.2 \%)$ & \\
\hline
\end{tabular}




\section{Prognostic significance of PD-Ll expression in melanoma}

Kaplan-Meier analysis was performed to evaluate patient survival curves according to PD-L1 and CD8+TIL status. In Kaplan-Meier analysis, there was no significant difference in overall survival (OS) depending on the PD-L1 expression status in TCs or TILs using both antibodies. When evaluated according to the MEL scoring system, PD-L1 (22C3) expression status was also not significantly correlated with patient prognosis $(P=0.793)$. Although the CD8+TIL high group showed a tendency toward more favorable OS than the CD8+TIL low group, this result did not reach statistical significance $(P=0.117)$. We additionally analyzed the survival differences depending on the combined status of PD-L1 (22C3) and CD8+TIL. Patients with PD-L1(22C3)-/ CD8+TIL high and PD-L1(22C3)+/CD8+TIL high showed a significantly more favorable OS, whereas the
PD-L1(22C3)+/CD8+TIL low group was associated with the worst clinical outcome $(P=0.039)$. Furthermore, a distinct survival difference was maintained when the PD-L1(22C3)+/CD8+TIL low subgroup was compared to the others $(P=0.010)$. PD-L1 (E1L3N) expression also showed no significant association with OS $(P=0.355$; Figure $3 C)$. However, similar results were obtained to those using the $22 \mathrm{C} 3$ clone in combined analysis, in which the survival of patients with PD-L1(E1L3N)-/CD8+TIL high and PD-L1(E1L3N)+/CD8+TIL high expression was significantly more favorable, while the PD-L1(E1L3N)+/CD8+TIL low group showed the worst clinical outcome $(P<0.001)$ (Figure 3$)$.

However, multivariate Cox proportional hazards analysis showed that PD-L1+/CD8+TIL low was not an independent prognostic factor in melanoma patients (Table 3 ).

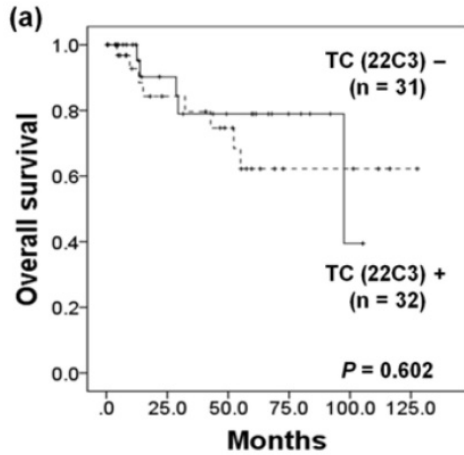

(d)

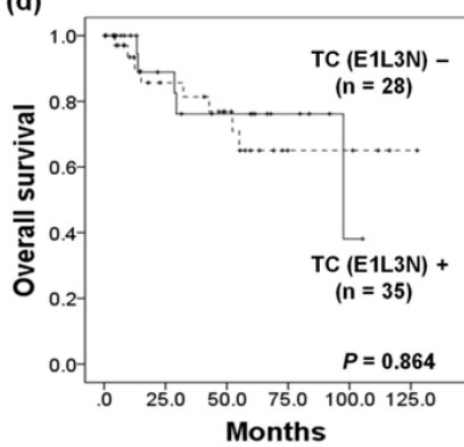

(g)

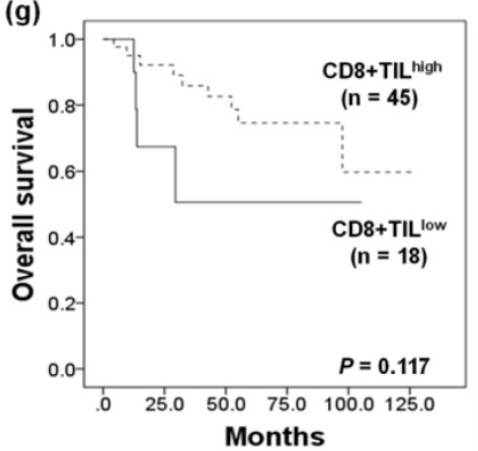

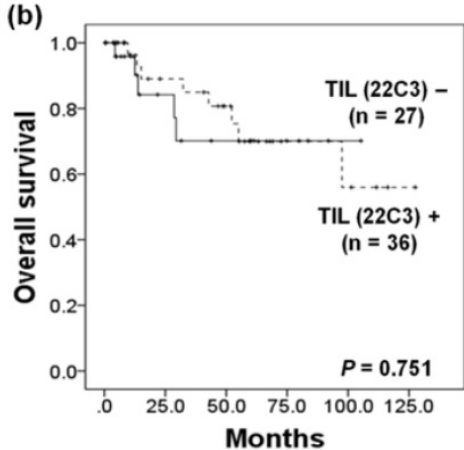

(e)

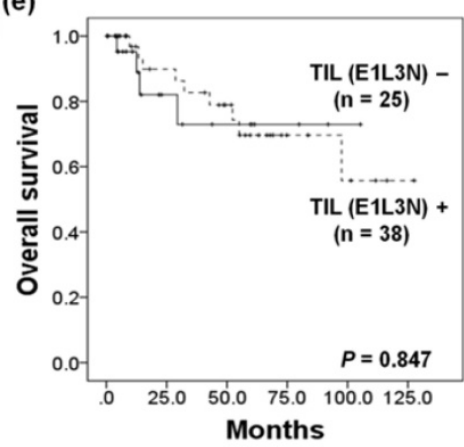

(h)

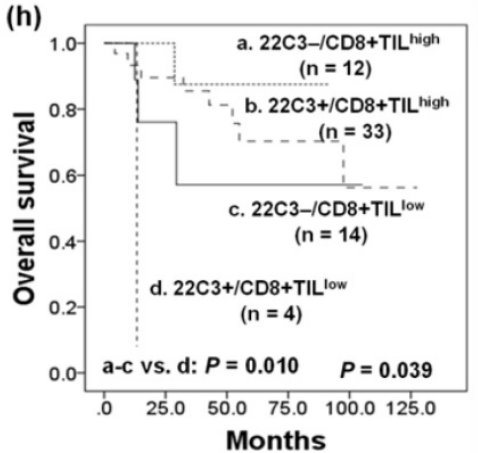

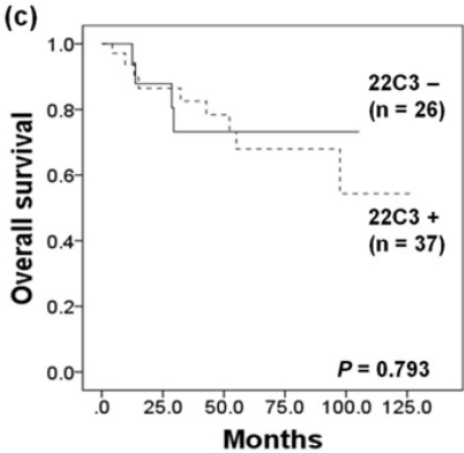

(f)

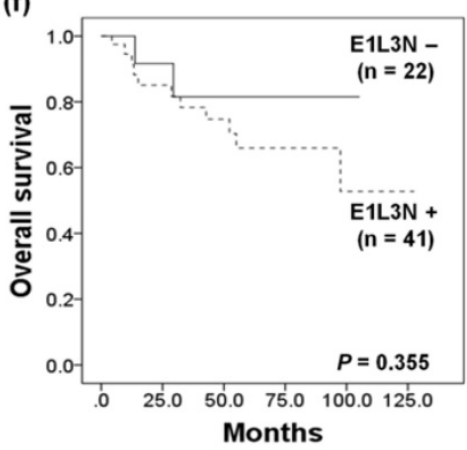

(i)

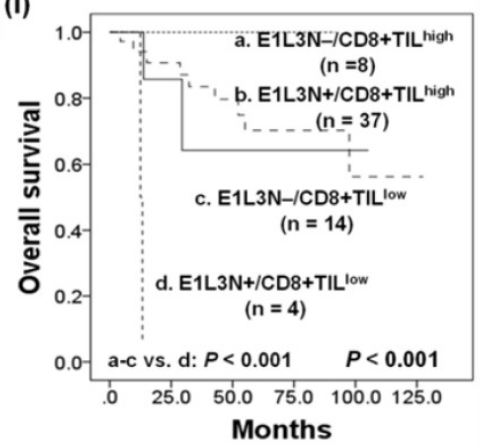

Figure 3. Kaplan-Meier survival curves of patients with (a) programmed death ligand 1 (PD-L1) expression (22C3) in tumor cells (TCs), (b) in tumor infiltrating lymphocytes (TILs), (c) in TCs and/or TILs (MEL), (d) PD-L1 expression (EIL3N) in TCs, (e) in TILs, (f) in TCs and/or TILs (MEL), (g) CD8+ TIL status, (h) combined status of PD-L1 (22C3) expression (MEL) and CD8+TILs, and (i) combined status of PD-L1 (EIL3N) expression (MEL) and CD8+TILs. 
Table 3. Multivariate Cox proportional hazard models for the predictors of overall survival in patients with melanoma

\begin{tabular}{|c|c|c|c|c|c|c|}
\hline \multirow[t]{2}{*}{ Factors } & \multicolumn{3}{|c|}{ Univariate analysis } & \multicolumn{3}{|c|}{ Multivariate analysis } \\
\hline & $\begin{array}{l}\mathrm{HR} \\
\end{array}$ & $95 \% \mathrm{CI}$ & $P$ & HR & $95 \% \mathrm{CI}$ & $P$ \\
\hline Age & 2.260 & $0.620-8.233$ & 0.217 & - & - & - \\
\hline Sex & 0.200 & $0.054-0.743$ & 0.016 & 0.071 & $0.010-0.531$ & 0.010 \\
\hline Melanoma subtype & 5.220 & $1.156-23.576$ & 0.032 & 12.056 & $1.067-136.265$ & 0.044 \\
\hline $\mathrm{cN}$ stage & 5.310 & $1.695-16.638$ & 0.004 & 0.548 & $0.032-9.285$ & 0.677 \\
\hline $\mathrm{cM}$ stage & 7.455 & $2.367-23.481$ & 0.001 & 14.119 & $2.620-76.073$ & 0.002 \\
\hline Lymphovascular invasion & 5.496 & $1.662-18.177$ & 0.005 & 152.891 & $11.363-2057.214$ & 0.044 \\
\hline Ulceration & 0.721 & $0.130-3.985$ & 0.708 & - & - & - \\
\hline Breslow thickness & 32.982 & $0.015-705.532$ & 0.372 & - & - & - \\
\hline$B R A F$ mutation & 0.789 & $0.214-2.910$ & 0.721 & - & - & - \\
\hline PD-L1(22C3)+/ CD8+TIL low & 11.134 & $1.119-110.742$ & 0.040 & 5.729 & $0.296-110.892$ & 0.248 \\
\hline PD-L1(E1L3N)+/ CD8+TIL low & 22.308 & $3.031-164.170$ & 0.002 & 6.768 & $0.330-138.628$ & 0.215 \\
\hline
\end{tabular}

$\mathrm{HR}$, hazard ratio; $\mathrm{CI}$, confidence interval.

\section{Discussion}

Although PD-L1/PD-1 inhibitors are already widely used in melanoma treatment, in-depth understanding of PD-L1 expression patterns among individual patients and subtypes is lacking, and the prognostic significance has not yet been elucidated. In fact, PD-L1 shows great significance as a predictor for response to an anti-PD-L1/PD-1 therapeutic agent rather than as a prognostic factor in other malignancies. Nevertheless, there are many reports suggesting PD-L1 as a prognostic factor in various cancers [25-30], although the prognostic impact of PD-L1 expression in melanoma is controversial. Previous studies reported that PD-L1 expression in TCs is not correlated with the survival of patients with melanoma [23, 31]. However, Ren et al. [19] reported that PD-L1 expression on TILs was an independent predictor of poor prognosis in patients with acral melanoma. Furthermore, Massi et al. [32] found that PD-L1 expression was an independent prognostic factor for BRAF inhibitor-treated melanoma patients. In contrast, Schaper-Gerhardt et al. [33] indicated that PD-L1 expression in the tumor tissue did not correlate with the outcome of BRAF inhibitor-based treatment. Therefore, further research on the roles and impact of PD-L1 in melanoma is required.

Recent studies indicated that PD-L1 expression correlated with the presence of oncogenic driver mutations. For example, PD-L1 expression was found to be significantly associated with EGFR-mutated non-small cell lung cancers [34], IDH-1-wild type glioblastomas [35], and BRAF-mutated colorectal cancers [36]. However, BRAF mutation status does not correlate with PD-L1 expression in melanoma [37], which was confirmed in the present study.

PD-L1-expressing TILs and TCs have important clinicopathological implications in various cancer types [38-44]. In urothelial carcinoma, analysis of PD-L1 (22C3 and SP263) expression in both TILs and TCs was used as a complementary diagnostic assay for anti-PD-1/PD-L1 treatment (pembrolizumab and durvalumab). Therefore, PD-L1 expression in TILs should not be overlooked. Notably, we adopted the MEL scoring system for determining the PD-L1 expression status in both TCs and TILs, which is based on modified criteria from a scoring system for hormone receptor status in breast cancer and was used in a recent clinical trial of melanoma [21].

IFN-Y is an essential inflammatory cytokine and is considered one of the most significant regulator of PD-L1 expression. Previous studies have shown that activated CD8+T cells are the major source of IFN-r production [45-48]. Notably, Tumeh et al. suggested that the type of TILs, particularly CD8+ cytotoxic T cells, is important for the action of immune checkpoint inhibitors [46]. Considering the importance of CD8+TILs in tumor immune microenvironment, we assessed correlation between CD8+ TILs and PD-L1 expression in our study.

TILs tend to be associated with a favorable prognosis in several types of cancer, including melanoma [23, 32, 49]. Moreover, Teng et al. [50] suggested that cancer can be categorized into four distinct subgroups, based on the status of PD-L1 and TILs. Melanoma patients with PD-L1+/TIL high (type I group) are considered to have adaptive immune resistance, and PD-L1-/TIL high (type IV group) seems to induce immune tolerance via the non-PD-1/PD-L1 pathway. PD-L1-/TIL low (type II group) is associated with immune ignorance. Finally, the rare PD-L1+/TIL low (type III group) phenotype is associated with oncogenic PD-L1 expression. In the present study, the combined status of PD-L1 and TILs, but not PD-L1 status alone, was significantly associated with clinical outcome. The PD-L1-expressing melanoma patients without TIL had the worst survival overall. However, PD-L1+/TIL low did not appear to be an independent factor for poor survival according to multivariate analysis, which might be due to the small number of patients. Although PD-L1+/TIL low was not an independent prognostic factor, combined analysis of both PD-L1 expression and TILs might predict survival differences in melanoma patients. 
In our study, PD-L1 expression in TILs (but not TCs) was observed in $50.8 \%$ of the cases. However, the survival difference according to PD-L1 positivity in TILs was not statistically significant (data not shown), in contrast to the results of Ren et al. [31]. This discrepancy can be explained by several factors. First, our study included patients with various subtypes of melanoma, not only the acral subtype. Second, unlike the previous study, we used the FDA-approved PD-L1 assay (22C3).

Since different expression patterns of PD-L1 have been reported according to the type of antibody used, we compared the detection rates with two antibodies (22C3 and E1L3). Although other researchers showed that different PD-L1 antibodies can produce varying positivity rates [51, 52], we found a high degree of concordance between $22 \mathrm{C} 3$ and E1L3N, in line with a previous study [53].

A relatively small patient population in a single institute, heterogeneous histological subtypes, and evaluation of a small tumor section of TMA are some of the limitation of this study. PD-L1-expressing melanoma patients without TILs seem to be only identified in a very small population (about 1\%) [50]. In the present study, only four cases were identified as PD-L1+/CD8+TIL low. Due to limited number of cases in each group, including the PD-L1+/CD8+TIL low group, we could not be certain of the prognostic impact of PD-L1 and TIL status using multivariate analysis. Thus, further studies are needed in a larger cohort of melanoma patients, based on the race and histological subtype, to validate the prognostic significance of combined status of PD-L1 and TILs. Nevertheless, our results obtained using the MEL scoring may support the prognostic value of PD-L1 expression in melanoma patients.

In conclusion, we found that the combined status of PD-L1 and TILs was associated with survival outcome in Korean melanoma patients, although it cannot act as an independent prognostic factor. Our results suggest that an integrated analysis of both PD-L1 and TILs might be useful for predicting prognosis in patients with melanoma.

\section{Abbreviations}

FDA: Food and Drug Administration; HPF: high-power field; IHC: immunohistochemistry; MEL: melanoma scoring system; PCR: polymerase chain reaction; PD-L1: Programmed death ligand 1; PD-1: Programmed death 1; TIL: tumor infiltrating lymphocytes; TMA: tissue microarray.

\section{Supplementary Material}

Supplementary tables.

http://www.jcancer.org/v10p3070s1.pdf

\section{Acknowledgments}

This research was funded by the Seoul National University Bundang Hospital research fund (grant number: 02-2016-017).

\section{Competing Interests}

The authors have declared that no competing interest exists.

\section{References}

1. Oh CM, Cho H, Won YJ, Kong HJ, Roh YH, Jeong KH, et al. Nationwide Trends in the Incidence of Melanoma and Non-melanoma Skin Cancers from 1999 to 2014 in South Korea. Cancer research and treatment: official journal of Korean Cancer Association. 2017.

2. Siegel RL, Miller KD, Jemal A. Cancer Statistics, 2017. CA: a cancer journal for clinicians. 2017; 67: 7-30.

3. Crombie IK. Racial differences in melanoma incidence. Br J Cancer. 1979; 40: 185-93.

4. Wang Y, Zhao Y, Ma S. Racial differences in six major subtypes of melanoma: descriptive epidemiology. BMC Cancer. 2016; 16: 691.

5. Kaunitz GJ, Cottrell TR, Lilo M, Muthappan V, Esandrio J, Berry S, et al. Melanoma subtypes demonstrate distinct PD-L1 expression profiles. Laboratory investigation; a journal of technical methods and pathology. 2017; 97: 1063-71.

6. Jung HJ, Kweon SS, Lee JB, Lee SC, Yun SJ. A clinicopathologic analysis of 177 acral melanomas in Koreans: relevance of spreading pattern and physical stress. JAMA dermatology. 2013; 149: 1281-8.

7. Lee SJ, Lim HJ, Choi YH, Chang YH, Lee WJ, Kim DW, et al. The clinical significance of tumor-infiltrating lymphocytes and microscopic satellites in acral melanoma in a korean population. Annals of dermatology. 2013; 25: 61-6.

8. Shain AH, Bastian BC. From melanocytes to melanomas. Nature reviews Cancer. 2016; 16: 345-58.

9. Madore J, Vilain RE, Menzies AM, Kakavand H, Wilmott JS, Hyman J, et al. PD-L1 expression in melanoma shows marked heterogeneity within and between patients: implications for anti-PD-1/PD-L1 clinical trials. Pigment cell \& melanoma research. 2015; 28: 245-53.

10. Gandini S, Massi D, Mandala M. PD-L1 expression in cancer patients receiving anti PD-1/PD-L1 antibodies: A systematic review and meta-analysis. Critical reviews in oncology/hematology. 2016; 100: 88-98.

11. Robert C, Long GV, Brady B, Dutriaux C, Maio M, Mortier L, et al. Nivolumab in previously untreated melanoma without BRAF mutation. The New England journal of medicine. 2015; 372: 320-30.

12. Beaver JA, Theoret MR, Mushti S, He K, Libeg M, Goldberg K, et al. FDA Approval of Nivolumab for the First-Line Treatment of Patients with BRAF(V600) Wild-Type Unresectable or Metastatic Melanoma. Clinical cancer research : an official journal of the American Association for Cancer Research. 2017; 23: 3479-83.

13. Mahoney KM, Freeman GJ, McDermott DF. The Next Immune-Checkpoint Inhibitors: PD-1/PD-L1 Blockade in Melanoma. Clinical therapeutics. 2015; 37: 764-82.

14. Frydenlund N, Mahalingam M. PD-L1 and immune escape: insights from melanoma and other lineage-unrelated malignancies. Human pathology. 2017; 66: 13-33.

15. Chen $\mathrm{YB}, \mathrm{Mu} \mathrm{CY}$, Huang JA. Clinical significance of programmed death-1 ligand-1 expression in patients with non-small cell lung cancer: a 5-year-follow-up study. Tumori. 2012; 98: 751-5.

16. Shi SJ, Wang LJ, Wang GD, Guo ZY, Wei M, Meng YL, et al. B7-H1 expression is associated with poor prognosis in colorectal carcinoma and regulates the proliferation and invasion of HCT116 colorectal cancer cells. PloS one. 2013; 8: e76012.

17. Muenst S, Schaerli AR, Gao F, Daster S, Trella E, Droeser RA, et al. Expression of programmed death ligand 1 (PD-L1) is associated with poor prognosis in human breast cancer. Breast cancer research and treatment. 2014; 146: 15-24.

18. Wei $\mathrm{W}, \mathrm{Xu} \mathrm{B}$, Wang $\mathrm{Y}, \mathrm{Wu} \mathrm{C}$, Jiang J, Wu C. Prognostic significance of circulating soluble programmed death ligand-1 in patients with solid tumors: A meta-analysis. Medicine. 2018; 97: e9617.

19. Ren M, Dai B, Kong YY, Lv JJ, Cai X. PD-L1 expression in tumor infiltrating lymphocytes is a poor prognostic factor for primary acral melanoma patients. Histopathology. 2018.

20. Taube JM, Anders RA, Young GD, Xu H, Sharma R, McMiller TL, et al. Colocalization of inflammatory response with B7-h1 expression in human melanocytic lesions supports an adaptive resistance mechanism of immune escape. Science translational medicine. 2012; 4: 127ra37.

21. Daud AI, Wolchok JD, Robert C, Hwu WJ, Weber JS, Ribas A, et al. Programmed Death-Ligand 1 Expression and Response to the Anti-Programmed Death 1 Antibody Pembrolizumab in Melanoma. Journal of clinical oncology: official journal of the American Society of Clinical Oncology. 2016; 34: 4102-9. 
22. Lee HS, Cho SB, Lee HE, Kim MA, Kim JH, Park DJ, et al. Protein expression profiling and molecular classification of gastric cancer by the tissue array method. Clinical cancer research: an official journal of the American Association for Cancer Research. 2007; 13: 4154-63.

23. Kakavand H, Vilain RE, Wilmott JS, Burke H, Yearley JH, Thompson JF, et al. Tumor PD-L1 expression, immune cell correlates and PD-1+ lymphocytes in sentinel lymph node melanoma metastases. Modern pathology: an official journal of the United States and Canadian Academy of Pathology, Inc. 2015; 28: 1535-44.

24. Nam SK, Yun S, Koh J, Kwak Y, Seo AN, Park KU, et al. BRAF, PIK3CA, and HER2 Oncogenic Alterations According to KRAS Mutation Status in Advanced Colorectal Cancers with Distant Metastasis. PloS one. 2016; 11: e0151865.

25. Wang Q, Liu F, Liu L. Prognostic significance of PD-L1 in solid tumor: An updated meta-analysis. Medicine. 2017; 96: e6369.

26. Wu P, Wu D, Li L, Chai Y, Huang J. PD-L1 and Survival in Solid Tumors: A Meta-Analysis. PloS one. 2015; 10: e0131403.

27. Xue S, Song G, Yu J. The prognostic significance of PD-L1 expression in patients with glioma: A meta-analysis. Scientific reports. 2017; 7: 4231.

28. Li X, Li M, Lian Z, Zhu H, Kong L, Wang P, et al. Prognostic Role of Programmed Death Ligand-1 Expression in Breast Cancer: A Systematic Review and Meta-Analysis. Targeted oncology. 2016; 11: 753-61.

29. Sun JM, Zhou W, Choi YL, Choi SJ, Kim SE, Wang Z, et al. Prognostic Significance of PD-L1 in Patients with Non-Small Cell Lung Cancer: A Large Cohort Study of Surgically Resected Cases. Journal of thoracic oncology: official publication of the International Association for the Study of Lung Cancer. 2016; 11: 1003-11.

30. Furue M, Kadono T. Melanoma therapy: Check the checkpoints. The Journal of dermatology. 2016; 43: 121-4.

31. Ren M, Dai B, Kong YY, Lv JJ, Cai X. PD-L1 expression in tumour-infiltrating lymphocytes is a poor prognostic factor for primary acral melanoma patients. Histopathology. 2018.

32. Massi D, Brusa D, Merelli B, Falcone C, Xue G, Carobbio A, et al. The status of PD-L1 and tumor-infiltrating immune cells predict resistance and poor prognosis in BRAFi-treated melanoma patients harboring mutant BRAFV600. Annals of oncology: official journal of the European Society for Medical Oncology. 2015; 26: 1980-7.

33. Schaper-Gerhardt K, Okoye S, Herbst R, Ulrich J, Terheyden P, Pfohler C, et al. PD-L1 status does not predict the outcome of BRAF inhibitor therapy in metastatic melanoma. European journal of cancer (Oxford, England: 1990). 2018; 88: 67-76.

34. Azuma K, Ota K, Kawahara A, Hattori S, Iwama E, Harada T, et al. Association of PD-L1 overexpression with activating EGFR mutations in surgically resected nonsmall-cell lung cancer. Annals of oncology: official journal of the European Society for Medical Oncology. 2014; 25: 1935-40.

35. Lee KS, Lee K, Yun S, Moon S, Park Y, Han JH, et al. Prognostic relevance of programmed cell death ligand 1 expression in glioblastoma. Journal of neuro-oncology. 2018; 136: 453-61.

36. Rosenbaum MW, Bledsoe JR, Morales-Oyarvide V, Huynh TG, Mino-Kenudson M. PD-L1 expression in colorectal cancer is associated with microsatellite instability, BRAF mutation, medullary morphology and cytotoxic tumor-infiltrating lymphocytes. Modern pathology: an official journal of the United States and Canadian Academy of Pathology, Inc. 2016; 29: $1104-12$.

37. Rodic N, Anders RA, Eshleman JR, Lin MT, Xu H, Kim JH, et al. PD-L1 expression in melanocytic lesions does not correlate with the BRAF V600E mutation. Cancer immunology research. 2015; 3: 110-5.

38. Bellmunt J, Mullane SA, Werner L, Fay AP, Callea M, Leow JJ, et al. Association of PD-L1 expression on tumor-infiltrating mononuclear cells and overall survival in patients with urothelial carcinoma. Annals of oncology: official journal of the European Society for Medical Oncology. 2015; 26: 812-7.

39. Kim HR, Ha SJ, Hong MH, Heo SJ, Koh YW, Choi EC, et al. PD-L1 expression on immune cells, but not on tumor cells, is a favorable prognostic factor for head and neck cancer patients. Scientific reports. 2016; 6: 36956.

40. Kim KJ, Yang HK, Kim WH, Kang GH. Combined prognostic effect of PD-L1 expression and immunoscore in microsatellite-unstable advanced gastric cancers. Oncotarget. 2017; 8: 58887-902.

41. Koirala P, Roth ME, Gill J, Piperdi S, Chinai JM, Geller DS, et al. Immune infiltration and PD-L1 expression in the tumor microenvironment are prognostic in osteosarcoma. Scientific reports. 2016; 6: 30093.

42. Thompson RH, Dong H, Kwon ED. Implications of B7-H1 expression in clear cell carcinoma of the kidney for prognostication and therapy. Clinical cancer research : an official journal of the American Association for Cancer Research. 2007; 13: 709s-15s

43. Herbst RS, Soria JC, Kowanetz M, Fine GD, Hamid O, Gordon MS, et al. Predictive correlates of response to the anti-PD-L1 antibody MPDL3280A in cancer patients. Nature. 2014; 515: 563-7.

44. Irie RF, DeNunzio FD. Immunotherapy of melanoma: current status and prospects for the future. The Journal of dermatology. 1993; 20: 65-73.

45. de Araujo-Souza PS, Hanschke SC, Viola JP. Epigenetic control of interferon-gamma expression in CD8 T cells. Journal of immunology research. 2015; 2015: 849573

46. Tumeh PC, Harview CL, Yearley JH, Shintaku IP, Taylor EJ, Robert L, et al. PD-1 blockade induces responses by inhibiting adaptive immune resistance. Nature. 2014; 515: 568-71.
47. Erdag G, Schaefer JT, Smolkin ME, Deacon DH, Shea SM, Dengel LT, et al. Immunotype and immunohistologic characteristics of tumor-infiltrating immune cells are associated with clinical outcome in metastatic melanoma. Cancer research. 2012; 72: 1070-80.

48. Passarelli A, Mannavola F, Stucci LS, Tucci M, Silvestris F. Immune system and melanoma biology: a balance between immunosurveillance and immune escape. Oncotarget. 2017; 8: 106132-42.

49. Lee WJ, Lee YJ, Shin HJ, Won $\mathrm{CH}$, Chang SE, Choi JH, et al. Clinicopathological significance of tumor-infiltrating lymphocytes and programmed death-1 expression in cutaneous melanoma: a comparative study on clinical subtypes. Melanoma research. 2018.

50. Teng MW, Ngiow SF, Ribas A, Smyth MJ. Classifying Cancers Based on T-cell Infiltration and PD-L1. Cancer research. 2015; 75: 2139-45.

51. McLaughlin J, Han G, Schalper KA, Carvajal-Hausdorf D, Pelekanou V, Rehman J, et al. Quantitative Assessment of the Heterogeneity of PD-L1 Expression in Non-Small-Cell Lung Cancer. JAMA oncology. 2016; 2: 46-54.

52. Sheffield BS, Fulton R, Kalloger SE, Milne K, Geller G, Jones M, et al. Investigation of PD-L1 Biomarker Testing Methods for PD-1 Axis Inhibition in Non-squamous Non-small Cell Lung Cancer. The journal of histochemistry and cytochemistry: official journal of the Histochemistry Society. 2016; 64: 587-600.

53. Rimm DL, Han G, Taube JM, Yi ES, Bridge JA, Flieder DB, et al. A Prospective, Multi-institutional, Pathologist-Based Assessment of 4 Immunohistochemistry Assays for PD-L1 Expression in Non-Small Cell Lung Cancer. JAMA oncology. 2017; 3: 1051-8. 